\title{
Scientific Productivity of Researchers in Sarem Women's Hospital, Tehran, Iran (1987-2013)
}

\section{A R T I C L E I N F O}

\section{Article Type}

Original research

\section{Authors}

Karimi MansoorAbad E.* $M A$

How to cite this article Karimi MansoorAbad E. Scientific Productivity of Researchers in Sarem Women's Hospital, Tehran, Iran (1987-2013). Sarem Journal of Reproductive Medicine. 2018; 2(2):51-54.
*"Sarem Fertility \& Infertility Research Center (SAFIR)" and "Sarem Cell Research Center (SCRC)", Sarem Women's Hospital, Tehran, Iran

\section{Correspondence}

Address: Sarem Women's Hospital, Basij Square, Phase 3, Ekbatan Town, Tehran, Iran. Postal Code: 1396956111

Phone: +98 (21) 44670888

Fax: +98 (21) 44670432

karimi_elham84@yahoo.com

\section{Article History}

Received: February 14, 2017

Accepted: May 21, 2017

ePublished: June 15, 2018

\section{A B S T R A C T}

Aims Qualitative and quantitative analysis of the scientific works in educational and research organizations can result in recognition of the trends of production and development of scientific information and researches in different branches of sciences. The objective of this study was to investigate the scientific productivity of researchers in Sarem women's hospital, Tehran, Iran (1987-2013).

Materials \& Methods In this descriptive study, all Persian and English documents published by researchers of Sarem women's hospital from 1987 to 2013 were evaluated. Based on the extracted data from the research center of the hospital and ISI database, the frequency of scientific works produced each year were found according to the type of documents including journal article, book, conference paper and dissertation. The collaborative coefficient (CC) of the authors in the production of the scientific works was calculated and the data were analyzed using SPSS 22 software.

Findings A total of 168 scientific works were published by the researchers in Sarem women's hospital from 1987 to 2013 . There were 82 (48.8\%) journal articles and this type was the most abundant scientific works among different types of scientific documents. The highest amount of scientific works was published in 2012. The CC level of the researchers in the hospital was found as 0.72 .

Conclusion Scientific productivity of researchers in Sarem women's hospital shows increasing trend in recent years. Journal articles are the most abundant scientific works among different types of scientific documents in the hospital. There is a high level of collaborative coefficient for the researchers of the hospital.

Keywords Publications; Sarem Women's Hospital; Researchers; Manuscripts, madicalas, Topic; Books; Academin Dissertatins as Topic; Bibliometrics 


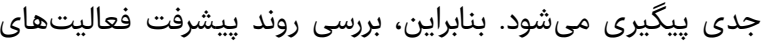

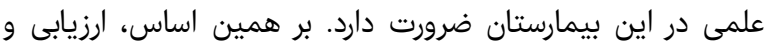

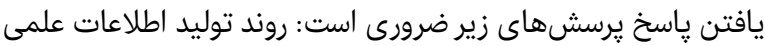

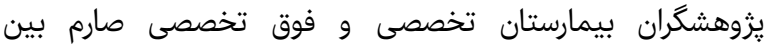

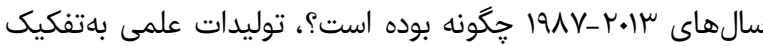

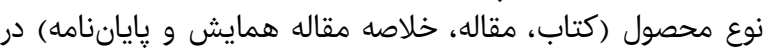

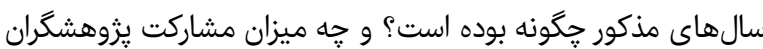

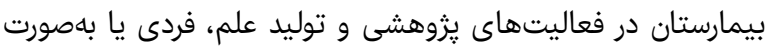

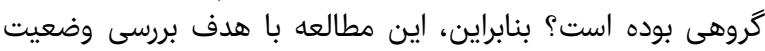

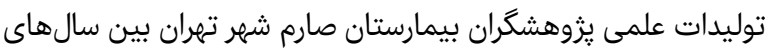

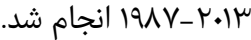

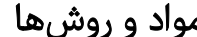

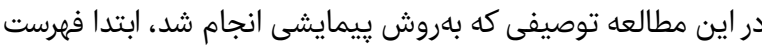

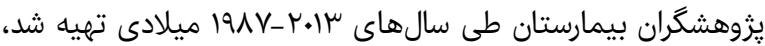

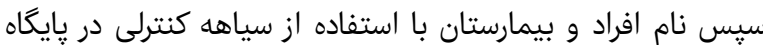

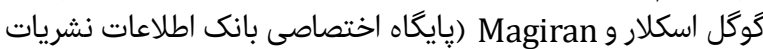

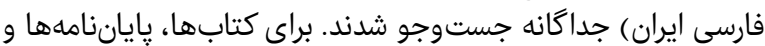

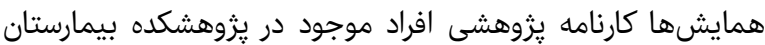

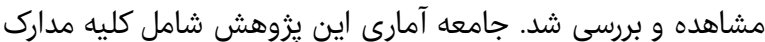

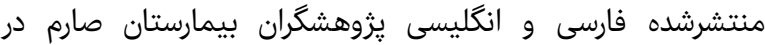

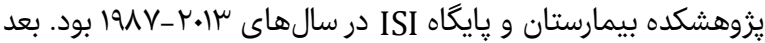

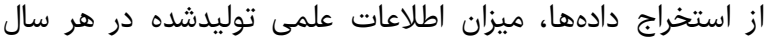

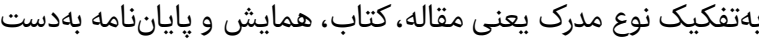

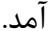

براى يافتن مقالات مرتبط با بيمارستان در پايكاهماى مذكور نام

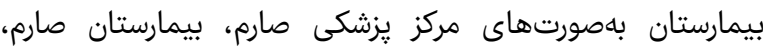

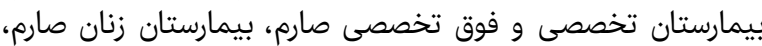
Sarem و Sarem Woman's Hospital ،Sarem Hospital Cell Research Center ضريب همكارى گروهى (CC) نويسندكان مطابق فرمول ا محاسبه

$$
C C=1-\frac{\sum_{j=1}^{k}\left(\frac{1}{j}\right) \times F_{j}}{N}
$$

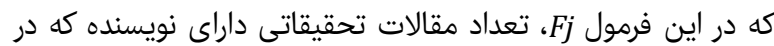

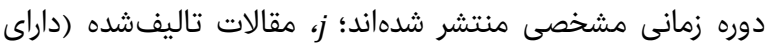

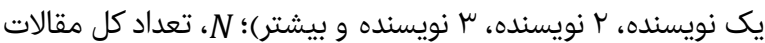

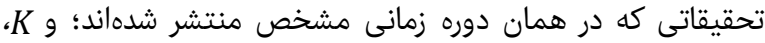

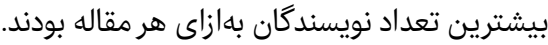

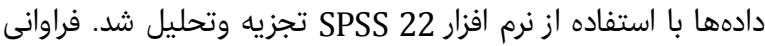

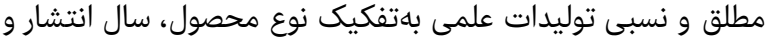

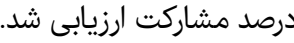

\section{يافتهها}

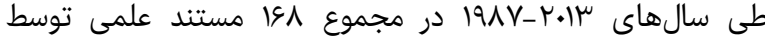

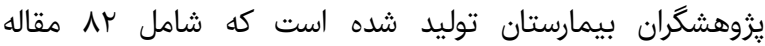

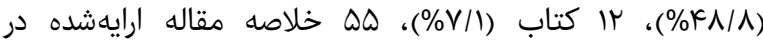

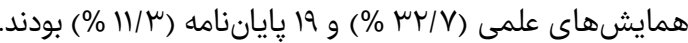

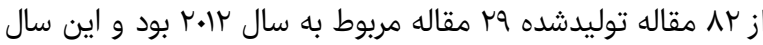

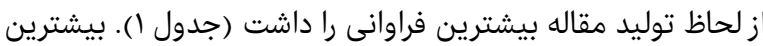

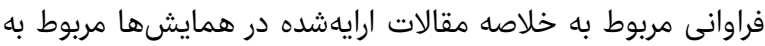

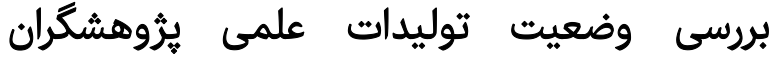

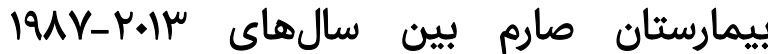

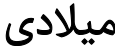

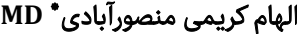

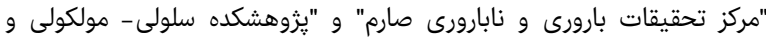
سلولهاى بنيادى صارم"، بيمارستان فوق تخصصى صارئ صارم، تهران، ايران

جكيده اهداف: تجزيه و تحليل كمى و كيفى فعاليتهاى علمى واحدهاى آموزشى و ور ورو

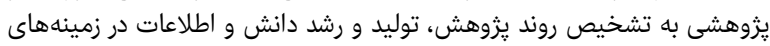

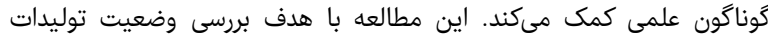

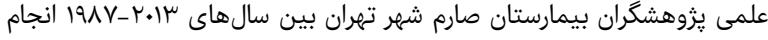

شد.

مواد و روشها: در اين مطالعه توصيفى كه بهروش ييمايشى انجام شد كليه

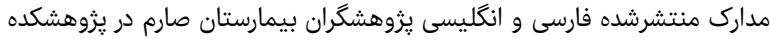

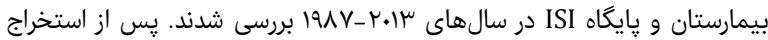

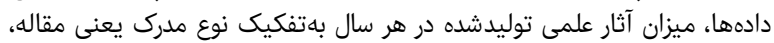

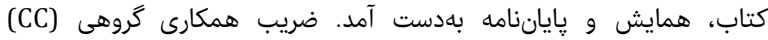

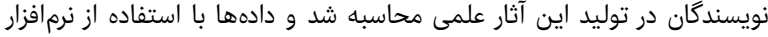
SPSS 22

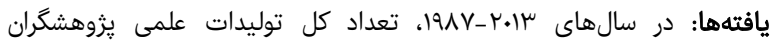

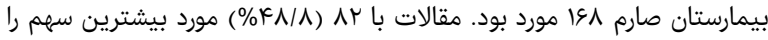

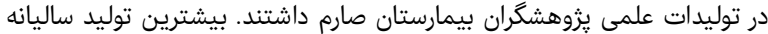

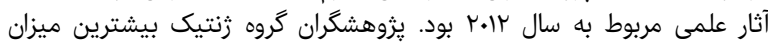

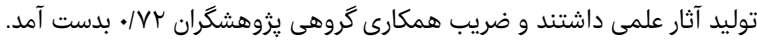

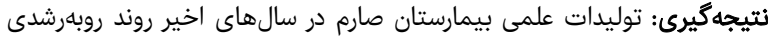

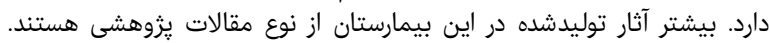

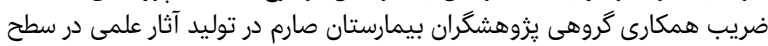

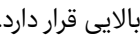

كليدواثهها: توليد علم، بيمارستان صارم، يزوهشكران، علمسنجى

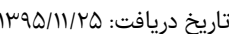

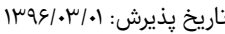

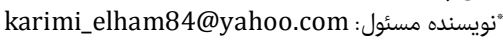

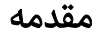

اطلاعات نقش عمدهاى در ييشرفت جوامع امروزى دارد و از بعد إزداء

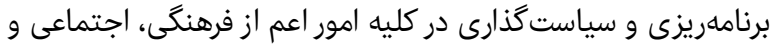

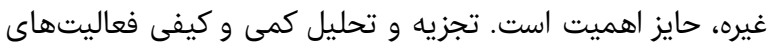

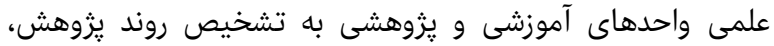

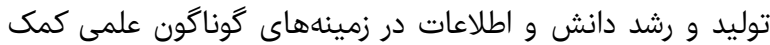

مىكند و در امور مختلف كاربرد دارد[1].

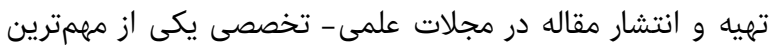

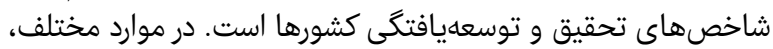

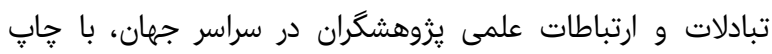

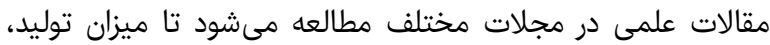

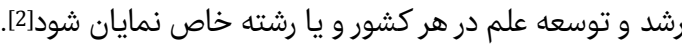

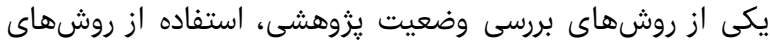

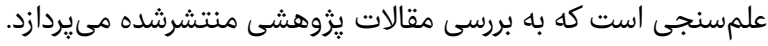

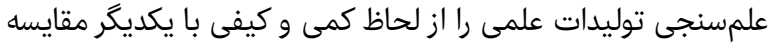

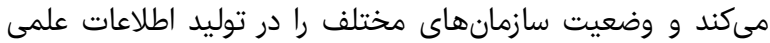
مشخص مىكند[2].

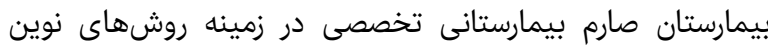

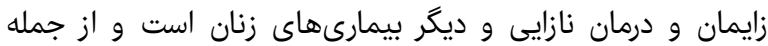

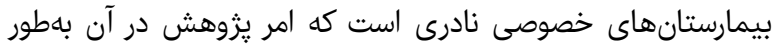




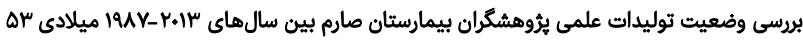

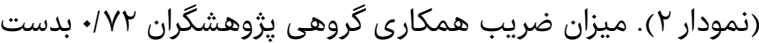

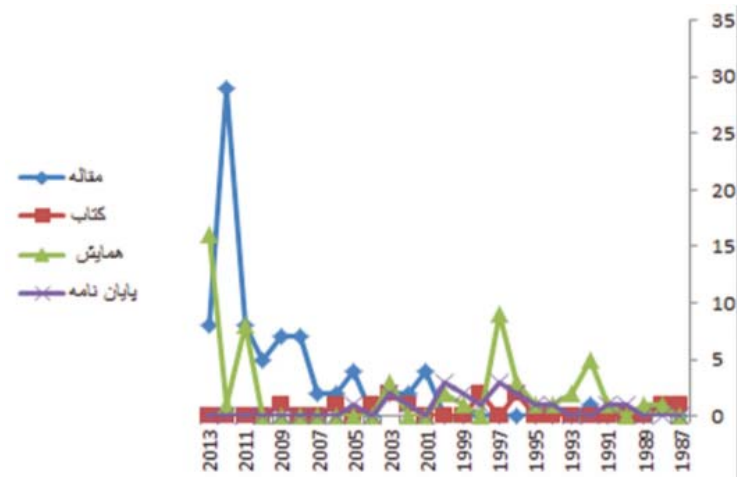

نمودار () روند كلى توليد اطلاعات علمى يزوهشگران بيمارستان صارم بهتفكيك

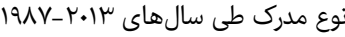

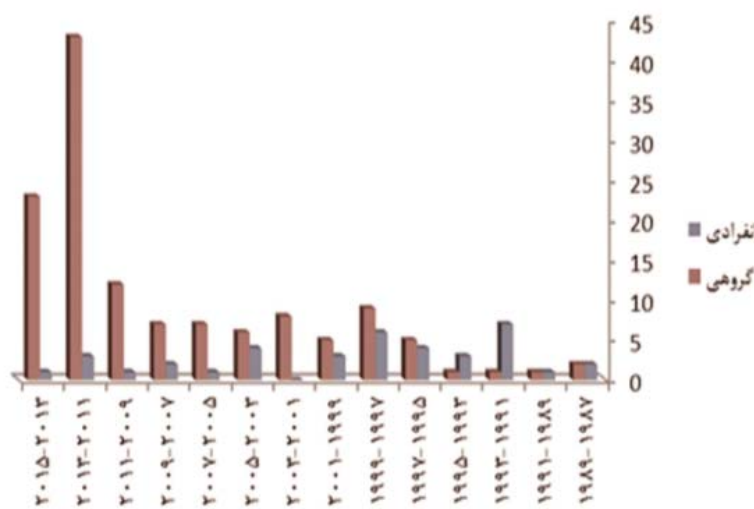

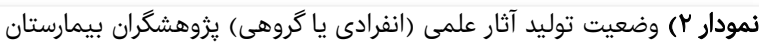

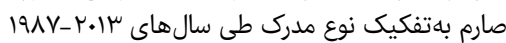

توليدات علمى مراكز علمى- آموزشى و يزوهشى و ارزيابى آنان

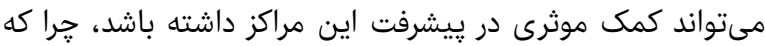

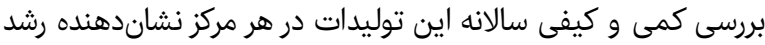

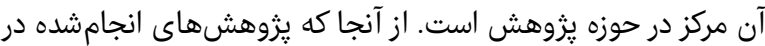

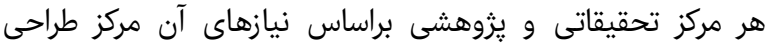

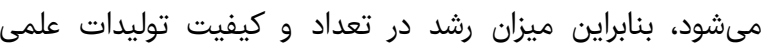

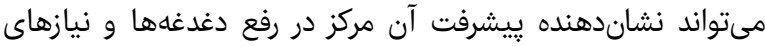

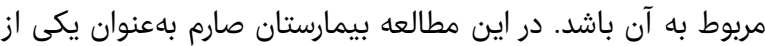

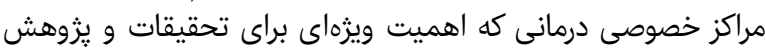

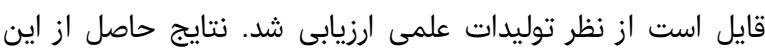

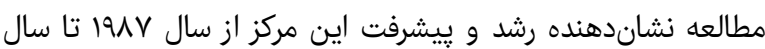

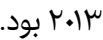

براساس مطالعات قبلى از جمله مطالعه اعظمى [3]، بذرافثشان و

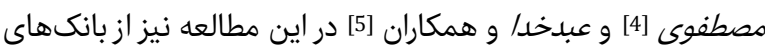

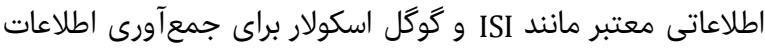

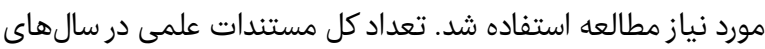

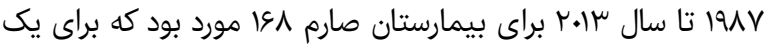

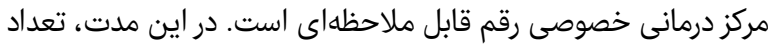

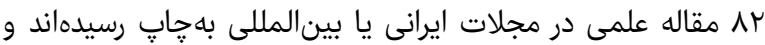

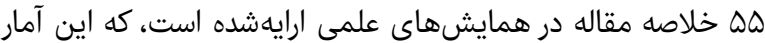

سال ساربr با تعداد ع| خلاصه مقاله بود و تعداد كتابهاى

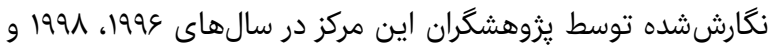

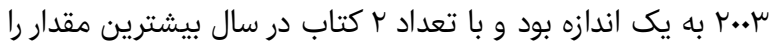

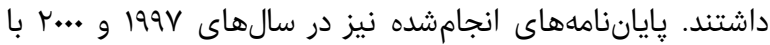

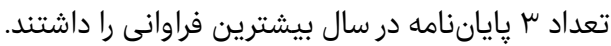

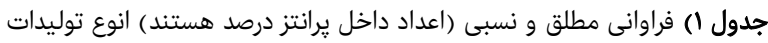

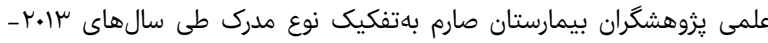

1919

\begin{tabular}{|c|c|c|c|c|c|}
\hline كل آثار & بايانانامه & همايش & كتاب & مقاله & سال \\
\hline$(\cdot / 8) 1$ & - & - & $\left(\Lambda /{ }^{\mu}\right) 1$ & - & 1971 \\
\hline$(1 / \Lambda)^{\mu}$ & - & $(1 / \wedge) 1$ & $(\Lambda / \mu) 1$ & $(1 / \Gamma) 1$ & 1941 \\
\hline$(\cdot 19) 1$ & 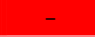 & $(1 / A) 1$ & - & - & 1919 \\
\hline$(\cdot \mid 9) 1$ & $\left(\Delta / \mu^{\mu}\right) 1$ & - & - & - & 199. \\
\hline$(1 / r) r$ & $\left(\Delta / \mu^{\mu}\right) 1$ & $(1 / A) 1$ & - & - & 1991 \\
\hline$(\mu / \varphi)^{\varphi}$ & - & $(9 / 1) \Delta$ & - & $(1 / T) 1$ & 1994 \\
\hline$(1 / r) r$ & - & $(\mu / \varphi)^{\mu}$ & - & - & 1994 \\
\hline$(I / r) r$ & $(\Delta / \mu) 1$ & $(1 / \wedge) 1$ & - & - & 1998 \\
\hline$(I / r) r$ & $\left.\left(\Delta / \mu^{\mu}\right)\right)$ & $(1 / A) !$ & - & - & $199 \mathrm{Q}$ \\
\hline$(K / K) V$ & $(1 \cdot / \Delta) r$ & $(\Delta / \Delta)^{\mu}$ & $(1 \& / V) r$ & - & 1998 \\
\hline$(V / I) \mathbb{I}$ & $(\mid \Delta / \Lambda)^{\mu}$ & $(1 / / 4)^{q}$ & - & - & 1991 \\
\hline$(1 / \Lambda)^{\mu}$ & $(\Delta / \mu) 1$ & - & $(1 \& / V) r$ & - & 1991 \\
\hline$(1 / \Lambda)^{\mu}$ & $(1 \cdot / \Delta) Y$ & $(1 / A) 1$ & - & - & 1999 \\
\hline$(\mu / \cdot) \Delta$ & $(\mid \Delta / \Lambda) \psi$ & $(\mu / \varphi) r$ & - & - & r... \\
\hline$(r / F)^{k}$ & - & - & - & $(k / q)^{k}$ & $r .1$ \\
\hline$(T / \mathcal{F})^{k}$ & $\left(\Delta /{ }^{\mu}\right) \mid$ & - & $(\Lambda / \mu) 1$ & $(r / K) r$ & $r . . r$ \\
\hline$(\Delta / \mathcal{F}) q$ & $(1 \cdot / \Delta) Y$ & $(\Delta / \Delta)^{\mu}$ & $(18 / V) r$ & $(r / F) r$ & $r . . \mu$ \\
\hline$(\cdot \mid 9) 1$ & - & - & $(\Lambda / \mu))$ & - & r...F \\
\hline$(\mu / \cdot) \Delta$ & $(\Delta / \mu) 1$ & - & - & $(k / q)^{k}$ & $r \cdots \Delta$ \\
\hline$(1 / \wedge)^{\mu}$ & - & - & $(\Lambda / \mu))$ & $(r / F) r$ & $r_{\ldots .}$ \\
\hline$(1 / r) r$ & - & - & - & $(r / F) r$ & $r . v V$ \\
\hline$(K / T) V$ & - & - & - & $(\Lambda / \Delta) V$ & $r_{* \cdots \Lambda}$ \\
\hline$(k / \Lambda) \wedge$ & - & - & $(\Lambda / \mu) !$ & $(\Lambda / \Delta) V$ & $r \ldots q$ \\
\hline$(\omega / \cdot) \Delta$ & - & - & - & $(\varphi / 1) \Delta$ & r.1. \\
\hline$(9 / \Delta) 18$ & - & $(\mid K / Q) \wedge$ & - & $(9 / 1) \wedge$ & $r \cdot 11$ \\
\hline$(\mathrm{IV} / q)^{\mu}$. & - & $(1 / \wedge) 1$ & - & $\left(\mu \Delta / \mu^{\mu}\right) r^{\prime}$ & r. \\
\hline$(\| F / \mu) K K$ & - & $(r q / 1) \mid q$ & - & $(9 / 1) \wedge$ & r.1\% \\
\hline$(1 . . / \cdot) 18 \Lambda$ & $(1 . .1 \cdot 0) 19$ & $(1 \ldots / \cdot) \Delta Q$ & $(1 . . / \cdot) \mathbb{R}$ & $(1 \% / \cdot) \wedge r$ & جمع \\
\hline
\end{tabular}

در بيمارستان صارم روند توليد آثار علمى طى سالهاى سرا. 19^V-Y

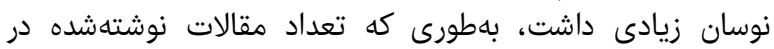

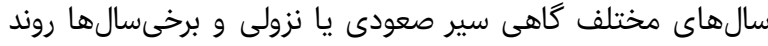

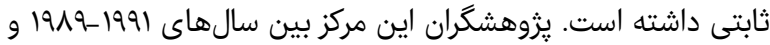

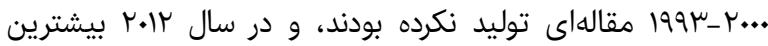

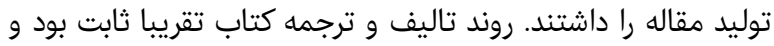

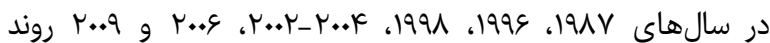

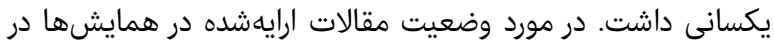

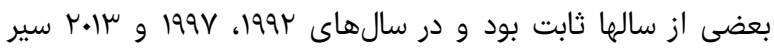

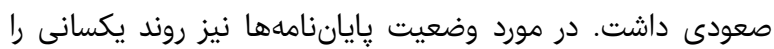

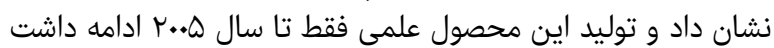

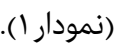

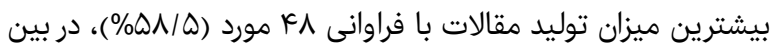

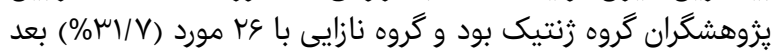

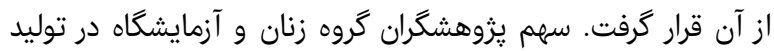

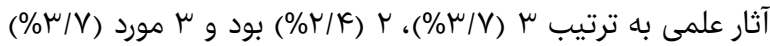

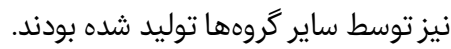

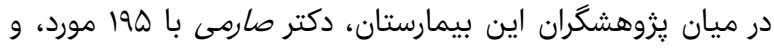

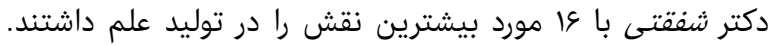

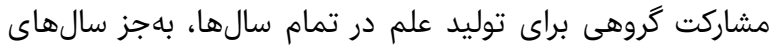

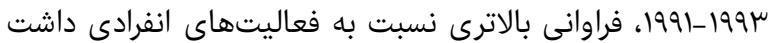


تاييديه اخلاقى: موردى توسط نويسنده گزارش نشده است.

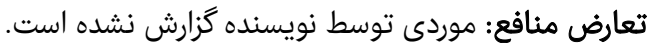

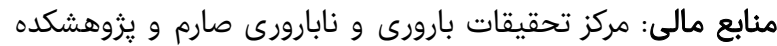

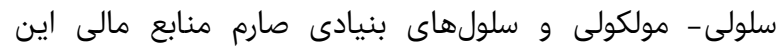

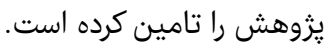

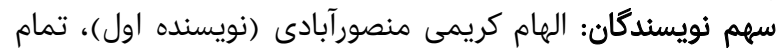

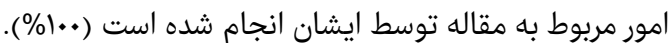

\section{منابع}

1- Osareh F, Sohieli F. A study using scientometric indicators on the scientific information produced by researchers of the water department of Khuzestan water and power authority during 1998-2006. Natl Stud librariansh Inf Organ. 2009;19(4):263-78. [Persian]

2- Sabaghinejad Z, Parsaei Mohammadi P, Baji F. Production trends of Middle Eastern countries in the field of nursing in Scopus base within the years 2010. In Press. [Persian]

3- Azami M. Review of the medical faculty participation in knowledge production during the period from 2000 to 2007 and factors affecting the web of science database. Natl Stud librariansh Inf Organ. 2010;13(3):1-5. [Persian]

4- Bazrafshan A, Mostafavi E. A scientometric overview of 36 yearsof scientific productivity by pasteurinstitute of Iran in ISI SCIE. J Health Adm. 2012;14(45):7-10. [Persian]

5- Abdekhoda H, Ghazi MirSaeed S, Nourzi A. Evaluation of scientific production of Iranian medical domain based on the document indexed from scientific journals in chosen databases, between 2005-2009. Payavard-eSalamat. 2010;4(1-2):18-30. [Persian]

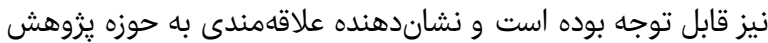

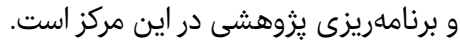

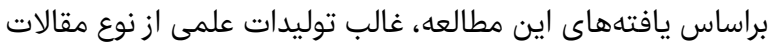

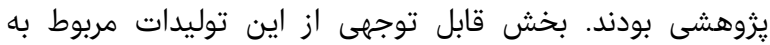

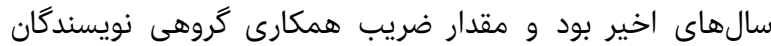

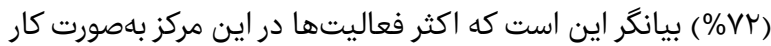

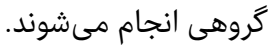

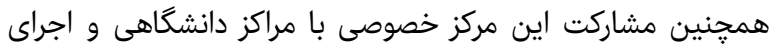

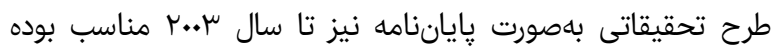

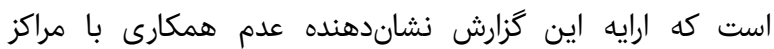

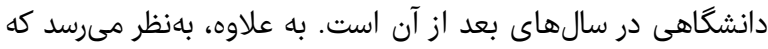

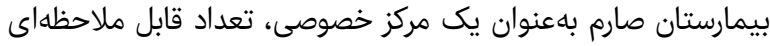
كتاب توليد كرده است. با توجه به اينكه ارزيابى دورهاى و وهندين ساله مراكز على علمى نقاط

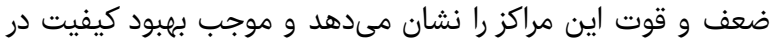

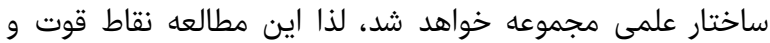
ضعف بيمارستان صارم در توليدات علمى خود در در سالهای

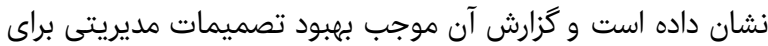
ارتقا كمى و كيفى محصولات علمى اين بيمارستان در سالهان آناى آينده خواهد شد. نتيجه گيرى توليدات علمى بيمارستان صارم در سالهاى اخير روند روبهرشدى

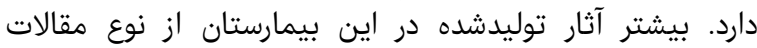

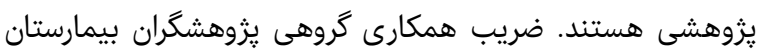
صارم در توليد آثار در سطح بالايى قرار دارد. تشكر و قدردانى: موردى توسط نويسنده گزارش نشده است. 